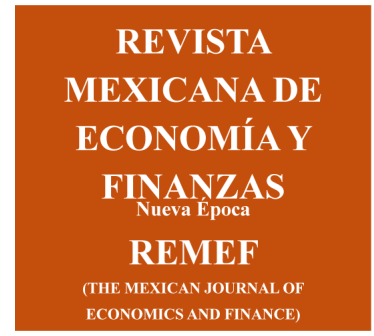

\begin{tabular}{c}
\hline Revista Mexicana de Economía y Finanzas \\
Nueva Época \\
Volumen 15 Número 2 , Abril - Junio 2020, pp. 227-240 \\
DOI: https://doi.org/10.21919/remef .v15i2.367 \\
(Primera recepción: 28/enero/2019, \\
última recepción: $15 /$ diciembre/2019; \\
aceptado: $21 /$ enero/2020)
\end{tabular}

\title{
Niveles de competencia en el mercado doméstico de transporte aéreo en Colombia y comparativa con nueve economías
}

\author{
Juan Carlos Garmendia Mora ${ }^{1}$ \\ Politécnico Grancolombiano, Colombia
}

\section{Resumen}

El objetivo fue estudiar el nivel de competencia que ha alcanzado el mercado doméstico del transporte aéreo en Colombia; se desarrolló un enfoque cuantitativo, tomando mediciones de las rutas principales del mercado colombiano; se calcularon los precio/Km y los niveles de concentración utilizando el índice Herfindahl-Hirschman (IHH); estas dos variables se correlacionaron y se contrastaron con los mercados domésticos de nueve países. El mercado doméstico colombiano tiene una alta concentración, Avianca es la aerolínea dominante con un $68,59 \%$ de las frecuencias; el IHH para el mercado colombiano es de 5.549, que al compararlo con el resto de países se ubica en una posición intermedia. Así mismo, el indicador precio/ $\mathrm{Km}$ del mercado colombiano está cercano al promedio de los diez países comparados. En cuanto a las limitaciones, la muestra de mercados se ampliará en una segunda etapa. No hay un estudio que mida los niveles de concentración del transporte aéreo doméstico en Colombia, utilizando el IHH y tomando como datos el número de frecuencias de vuelo. En Colombia se observa un equilibrio logrado con base a vigilancia de las autoridades competentes y cierto accionar prudente por parte de las aerolíneas.

Clasificación JEL: competencia, transporte aéreo, mercado, aerolíneas, impuestos y tasas

Palabras clave: A120, D430, M210, L130, L930

\section{Levels of competition in the domestic air transport market in Colombia}

\section{Abstract}

The objective was to study the level of competition that the domestic air transport market has reached in Colombia; a quantitative approach was developed, taking measurements of the main routes of the Colombian market; Price / Km and concentration levels were calculated using the Herfindahl - Hirschman (IHH) index; these two variables were correlated and contrasted with the domestic markets of nine countries. The colombian domestic market has a high concentration, Avianca is the dominant airline with $68.59 \%$ of frequencies; The HHI for the colombian market is 5,549, which when compared to other countries is located in an intermediate position. Likewise, the price / km indicator of the colombian market is close to the average of the ten countries compared. Regarding the limitations, the sample of markets will be expanded in a second stage. There is no study that measures the concentration levels of domestic air transport in Colombia, using the IHH and taking the number of flight frequencies as data. In Colombia there is a balance achieved based on vigilance of the competent authorities and some prudent action by the airlines.

JEL Classification: A120, D430, M210, L130, L930

Keywords: competition, air transport, markets, airlines, taxes and rates

\footnotetext{
${ }^{1}$ Email: juangarmendia@yahoo.com
} 


\section{Introducción}

Uno de los temas más importantes que se manejan en el entorno empresarial es el desarrollo de los mercados. Para Méndez (2014) el mercado es "el ámbito en que concurren oferentes y demandantes de bienes y servicios para llevar a cabo transacciones comerciales" (p.291). Así mismo señala Méndez que algunos de los elementos que constituyen el mercado son la oferta, la demanda y el precio. Se trata de alcanzar una meta que es difícil obtener y que cuando se consigue puede resultar corta su duración: el equilibrio del mercado. Existe una gran variedad de mercados de productos y servicios, los cuales se comportan de manera diversa, dependiendo de aspectos tales como el número de empresas oferentes que lo conforman, de acuerdo a su tamaño, los posibles bienes sustitutos, las tecnologías involucradas, las barreras de entrada, entre otros.

De acuerdo a las características de los mercados, los gobiernos intervienen en los mismos con el fin que los consumidores tengan un trato justo y correcto por parte de los oferentes, aunque estos objetivos no siempre se consigan. El mercado del transporte aéreo de pasajeros ha sido, desde sus inicios, uno de los más intervenidos y regulados por los entes competentes. Al respecto Cuevas (2009) indica:

Históricamente, el servicio de transporte aéreo y de carga ha sido considerado como un servicio público que debe ser regulado por el Estado... Con la introducción del Airline Deregulation Act en 1978, los Estados Unidos fueron pioneros en la desregulación del sector. A partir de 1983 las aerolíneas estadounidenses obtuvieron libertad para determinar las rutas, destinos, frecuencias y tarifas de los vuelos internos. La desregulación en el resto de países inició en la década de 1990 cuando se optó por privatizar aerolíneas y liberar tarifas y rutas (p.09)

Ciertamente, en los países latinoamericanos ha sido más lento el proceso de desregularización y liberalización de los mercados del transporte aéreo, muchas veces hecho de manera obligatoria, producto de la quiebra de las aerolíneas pertenecientes a los estados, o simplemente de las crisis económicas que se suscitaron a finales de los años 80 y principios de los 90 del siglo pasado.

Nauffal (2007) comenta que hasta 1997 se finalizó la desregularización del transporte aéreo en Europa. En el espacio latinoamericano, Colombia es hoy en día el tercer mercado doméstico más grande de América Latina, superado solo por Brasil y México. El mercado al que concurren las aerolíneas posee ciertas características particulares, tales como los costos de entrada prohibitivos, lo que reduce los incentivos a querer operar en este mercado; como segundo punto, la competencia dentro de este mercado no es tan fácil debido a que en algunos países todavía existen fuertes regulaciones al sector. En Colombia, las aerolíneas poseen libertad para fijar sus tarifas, de acuerdo a la resolución 904 de Aerocivil (2012) lo cual es un paso importante en la desregulación del sector, aunque siguen existiendo otros elementos, como la asignación de las franjas horarias, que son potestad de la autoridad aeronáutica, lo cual puede beneficiar a algún actor oferente del mercado, de acuerdo a la distribución que se haga de estas.

Los precios de mercado están determinados por el libre juego de la oferta y demanda, mientras que los precios controlados son establecidos por el gobierno para coordinar la realización de objetivos individuales y colectivos a través del mecanismo de planeación en un sistema económico (Ortega, 2011). Esta es la disyuntiva que se repite entre los gobernantes desde épocas inmemoriales, dejar que la mano invisible del mercado haga su trabajo y equilibre los precios, obteniendo el máximo beneficio posible para los consumidores y los productores, o intervenir en el mismo para que el lado más fuerte no se aproveche del lado más débil.

Esta desregulación que se ha hecho en el mercado doméstico colombiano pareciera haber favorecido la libre competencia, esto se puede evidenciar si comparamos las participaciones de mercado en tres momentos distintos: de acuerdo a Nauffal (2007) en la década de 1970. Avianca fue considerada un monopolio, ya que llegó a poseer hasta el $70 \%$ del mercado doméstico. Luego para el año 2015, según Pabón y otros 
(2015) (Pabón, Henao, Rodriguez, Campo, \& Herrera, 2015) en el mercado doméstico de aerolíneas, Avianca transportaba el $55,45 \%$ de los pasajeros, seguida por LAN Colombia con 18,9\% y Viva Colombia con 12,4\% en un universo de 12 aerolíneas. Ya en el presente, según datos de Aerocivil (2018), para el período enero a julio 2018, Avianca sigue siendo el líder del mercado nacional con un 53,40\% de los pasajeros en vuelos regulares, seguida por Latam Colombia con un 19,14\%, en tercer lugar se encuentra Viva Colombia con un 12,97\%, a continuación Easy Fly con un 4,93\% y en quinto lugar Satena con un 4,80\% dentro de un grupo de más de 12 aerolíneas. Entonces la distribución del mercado se ha hecho más equitativa, aunque todavía destaca la dominancia de Avianca.

Pero para muchos usuarios y ciudadanos en general, las condiciones actuales del mercado de transporte aéreo en Colombia distan mucho de ser las más optimas y justas para los clientes, es por eso que se presentan propuestas de leyes que buscan revertir el proceso de liberalización del mercado y devolver a la autoridad competente la regulación de las tarifas, entre otros aspectos. Jurídicamente, existen iniciativas como la interpuesta por el congresista Arroyave, representante al Congreso de la República de Colombia, quien citado por Semana (2018) indica que.${ }^{\mathrm{El}}$ proyecto busca poner en cintura a las aerolíneas. Vamos a reglamentar las tarifas y no vamos a permitir que se siga dando esa asignación aleatoria de los precios de los vuelos".

Visto este panorama, se justificó realizar el estudio para alcanzar el objetivo: Estudiar el nivel de competencia que ha alcanzado el mercado doméstico del transporte aéreo en Colombia.

\section{Estado del arte}

\subsection{Competencia, libertad, elección}

Novelo (2015) recuerda el teorema de Adam Smith sobre la mano invisible, que de acuerdo con este ha sido abusado en su interpretación por algunos estudiosos de la economía, pero que en esencia hace ponderación del egoísmo individual como causante de la riqueza colectiva. Estos son los preceptos de la libre competencia que promueven el ingreso al mercado de cualquier competidor para que los consumidores o demandantes de productos o servicios tengan mayores opciones de elección. Se asume que mientras más oferentes allá en un mercado, habrá menos probabilidades que se pongan de acuerdo para fijar precios, por lo que la competencia hará que los precios se reduzcan, acercándose a los costos de producción.

En este orden de ideas, es importante reflejar la diferencia entre libertad de elección y posibilidades de sustituir. Puede que, de acuerdo a las políticas gubernamentales, se promueva la libertad para que cualquier empresa pueda entrar al mercado a ofrecer sus productos o servicios, pero la realidad puede ser distinta al no haber suficientes competidores que permitan al consumidor elegir entre varias opciones. Como recuerda Rodríguez (2012), al consumidor tener libertad para escoger, debe también tener varias opciones, porque de lo contrario la libertad sirve de poco.

Similar a lo que ocurre en industrias como las telecomunicaciones, en donde existen grandes barreras de entrada y por lo tanto el estado debe velar por la no conformación de monopolios, algo parecido ocurre en el mercado del transporte aéreo, esto lo destaca la Oficina de Regulación Económica (2009):

El transporte aéreo se caracteriza por poseer un nivel alto de regulación por parte del estado. Esto se debe tanto a la presencia de altas economías de escala y barreras de entrada, como a la necesidad de garantizar la seguridad a los usuarios. La legislación colombiana establece que esta actividad es un servicio público esencial y en el contexto iberoamericano, se reconoce como un sector estratégico para el desarrollo y la integración de las naciones (p.14).

Normalmente, un vuelo no es sustituto de otro, ya que las ciudades destino no están lo suficientemente cerca como para considerarlo así. Al respecto Cardoso (2016) indica que "el transporte aéreo de pasajeros no 
cuenta con otros medios de transporte lo suficientemente cercanos, en precio y tiempos de recorrido, para ser considerados como posibles sustitutos". Esto es otro de los argumentos ampliamente utilizados para favorecer la intervención de la autoridad competente en el mercado aéreo.

\subsection{Niveles de Concentración}

Una herramienta útil para evaluar los niveles de competencia en el mercado aéreo colombiano, son los indicadores de concentración; diferentes entidades privadas y públicas a nivel mundial, que se dedican a promover la competencia en los distintos mercados, utilizan estos indicadores, tales como la ratio de concentración, el factor Herfindahl-Hirschman (HH), la participación de mercado significativa y otros que han ido surgiendo y adaptándose a alguna industria en particular. Pero advierte (Castañeda, 2007) que el uso de estos indicadores es referencial y debe acompañarse con análisis complementarios que permitan tener un panorama más completo para poder concluir acertadamente acerca del desempeño del mercado.

Lo anterior es importante, entendiendo que pudieran existir mercados con alta concentración, pero que a su vez son bastante competitivos. Tomando como elemento principal el factor HH, este "se utiliza para medir la concentración del mercado. Este índice es sugerido como un indicador de estructura de mercado, dado que tiene en cuenta tanto el número de competidores como su participación relativa en el mismo, y se calcula como la suma al cuadrado de la participación porcentual de las i-esima empresas en la industria" (Gutierrez \& Zamudio, 2008, págs. 4-5). El índice va desde 0 hasta 10.000, entendiendo que el más alto valor representa una industria monopolizada. Así mismo, de acuerdo a (Pinzón, 2011), un índice Herfindahl-Hirschman menor a 1.000 expresa baja concentración en el mercado analizado, entre 1000 y 1.800 una concentración media, superior a 1.800 refleja una alta concentración. Entre todo el abanico de indicadores existentes para medir la concentración o desigualdad en los mercados, se escogió este porque posee amplio uso y credibilidad, ayuda a visualizar el objetivo de la investigación y se puede usar con los datos disponibles.

El índice Herfindahl-Hirschman ha sido diseñado y muy utilizado para observar los mercados financieros y otras industrias afines, pero no hay ningún impedimento para utilizarlo en la industria del transporte aéreo, pero si es recomendable acompañarlo de otros análisis, por ejemplo, la comparación del mercado colombiano con distintos mercados domésticos de otros países. Para esta investigación se tomaron mediciones de otros mercados latinoamericanos como Argentina, Chile, Perú, México y Brasil, además de Estados Unidos de Norte América, Canadá, España y Francia.

\subsection{Concentración - Precios}

Para complementar el estudio, es factible tomar las mediciones de otros indicadores como el precio/kilómetro para las distintas rutas en observación y compararlas entre sí. Esta medición aporta información importante relacionada con la competencia que puede desarrollarse en cada mercado. Para Betancor, Gallego, González (2013) la variable principal para medir la competencia en una ruta aérea es el precio/kilómetro, es un indicador de competencia que homogeniza la variable precio considerando la distancia de la ruta. Así mismo, es necesario comparar las mediciones de este indicador en el mercado colombiano, con los demás mercados domésticos anteriormente nombrados, para visualizar un cuadro más amplio y referenciado.

En relación con elementos teóricos anteriormente expuestos, mientras menos concentración haya en los mercados, mayores serán las posibilidades que se dé una libre competencia y como consecuencia los precios de los boletos aéreos disminuyan. Entonces es importante determinar la correlación entre las variables concentración de mercados, representado por el índice HH y precio/Km. Para esto se hace un análisis de correlación entre estas dos variables, el cual según Lind (2015) posee dos etapas: primeramente se realiza un diagrama de dispersión para observar visualmente la relación entre las variables estudiadas, en segunda instancia se 
calcula el coeficiente de correlación, el cual otorga un dato cuantitativo que expresa el nivel de relación que poseen estas variables.

De acuerdo a Martínez (2012) el coeficiente de correlación r puede tomar valores entre -1 y 1. Si el valor es positivo, la correlación es directa, si el valor es negativo, la correlación es inversa. Cuando $\mathrm{r}=1$ existe una correlación perfecta, si $\mathrm{r}$ es menor que 1 y mayor que 0,90 es excelente la correlación, si se encuentra entre 0,90 y 0,80 existe una correlación aceptable, si el coeficiente está entre 0,80 y 0,60 la correlación es regular, es mínima la correlación cuando el coeficiente está ubicado entre 0,60 y 0,30; por debajo de 0,30 no existe correlación.

\section{Metodología}

La investigación está constituida por un proceso, etapas o secuencia de pasos que se van desarrollando para la obtención del objetivo de la misma. La presente investigación posee un enfoque cuantitativo. Según Hernández y Mendoza (2018), en el enfoque cuantitativo el investigador realiza diversos pasos tales como: planteamiento de un problema de estudio delimitado y concreto, revisión de la literatura, se recolectan datos numéricos que se analizan mediante procedimientos estadísticos, obteniendo la mayor cantidad de datos posibles, con lo cual se busca explicar y predecir los fenómenos investigados, detectando regularidades y relaciones causales entre distintos elementos; este enfoque utiliza la lógica y el razonamiento deductivo.

Este es un estudio correlacional, aunque también se trató de explicar los resultados obtenidos; se trata de evidenciar el nivel de competencia que ha logrado el mercado doméstico de transporte aéreo en Colombia, representado por el índice $\mathrm{HH}$ y algunos otros elementos subyacentes; este índice es correlacionado con la variable precio normalizada por el número de kilómetros de cada ruta; además estos valores se comparan con otros mercados para obtener una perspectiva más completa y poder dar explicaciones sustentadas al fenómeno observado. Para Hernández, Collado, Baptista (2014) los estudios correlacionales pretenden establecer cómo se vinculan diversos elementos entre sí; en esta investigación se establece la concordancia de los niveles de concentración del mercado y el precio/Km; así mismo, los estudios explicativos están destinados a responder por las causas y en qué condiciones se manifiesta el fenómeno observado.

Así mismo, el diseño de la investigación es no experimental, con enfoque transversal en el entendido que sólo se observa el fenómeno en su contexto natural, para luego estudiar los datos aportados, analizarlos y visualizarlos, sin manipular ningún factor o variable en observación (Hernández y Mendoza, 2018). En la figura 1 se observa el flujograma que representa los pasos seguidos para el tratamiento de los datos; se tomaron las mediciones en dos momentos específicos de tiempo, con la intención de describir el fenómeno en esos instantes.

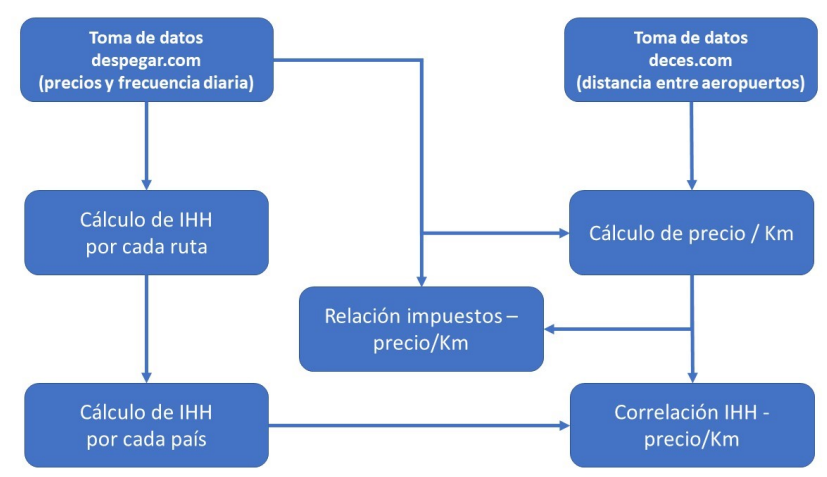

Figura 1. Proceso de recolección y procesamiento de datos Fuente: elaboración propia 
En este estudio, la unidad de análisis son las rutas troncales del mercado colombiano, recordando que cada ruta debe ser considerada como un mercado independiente; se levantó la información de todas las rutas troncales, es decir, el procedimiento fue censal, ya que de acuerdo a Galbiati (2016), la población es la totalidad de los elementos del conjunto que se quieren estudiar y el censo es la observación de la población completa. De manera similar, se levantó la información de las rutas troncales en México, Argentina, Chile, Perú, Brasil, España, Francia y Canadá; en Estados Unidos, por ser un mercado mucho más grande, no se pudieron tomar todas las rutas troncales, pero igualmente se revisó una muestra de 6 rutas principales, tanto en la costa este, como la costa oeste, así como la ruta Nueva York - Los Ángeles, que une las dos costas, esto con la intención de que sirviera de referencia o benchmarking, dado que este país tiene unos niveles de liberalización en el mercado del transporte aéreo que posiblemente no existe en ninguna otra nación; todas las rutas estudiadas se muestran en la tabla 1.

Por cada una de las empresas que operan cada ruta, se recogieron los siguientes datos: frecuencia diaria, precio mínimo y máximo para clase económica; este precio se discriminó mediante tres elementos que lo conforman: tarifa aerolínea, impuestos y tasas y los cargos por servicios. Además se indagó la distancia en kilómetros de cada una de estas rutas. La información de los precios y frecuencias de vuelo se recolectó de la plataforma especializada en venta de boletos aéreos despegar.com el 26 de septiembre de 2018, realizando las búsquedas para vuelos a realizarse el 11 de octubre; el segundo momento de búsqueda fue el 09 de noviembre de 2018 para vuelos con fecha 28 de noviembre de 2018. Los datos de distancia entre aeropuertos se obtuvieron del Directorio Cartográfico de España y América (DICES, 2018) el cual posee información confiable al respecto.

Con los datos recolectados se procedió a calcular el precio por kilómetro, discriminando el precio del boleto, los impuestos y tasas y los cargo por servicio. Se calculó también el índice Herfindahl-Hirschman por cada ruta; este índice se computó tomando como dato de concentración de cada aerolínea, el número de frecuencias diarias que poseían en esa ruta. Luego se calculó un promedio ponderado por cada país, para tener un elemento de comparación entre los países en estudio, de acuerdo a la siguiente formula:

$$
I H H=\sum_{i=1}^{n}\left(\frac{X_{i}}{X} 100\right)^{2}
$$

En donde i es el número de aerolíneas operando en la ruta en estudio, $X_{i}$ la frecuencia de vuelos de la iésima aerolínea y $X$ el total de frecuencia.

Tabla 1. Rutas estudiadas por cada país

\begin{tabular}{|c|c|c|c|}
\hline País & Rutas & País & Rutas \\
\hline Colombia & $\begin{array}{l}\text { Bogotá - Medellín } \\
\text { Bogotá - Cali } \\
\text { Bogotá - Barranquilla } \\
\text { Bogotá - Cúcuta } \\
\text { Bogotá - Cartagena } \\
\text { Bogotá - Bucaramanga }\end{array}$ & Chile & $\begin{array}{l}\text { Santiago - Antofagasta } \\
\text { Santiago - Puerto Montt } \\
\text { Santiago - Concepción } \\
\text { Santiago - Punta Arenas } \\
\text { Santiago - La Serena }\end{array}$ \\
\hline México & $\begin{array}{l}\text { CDMX - Cancún } \\
\text { CDMX - Guadalajara } \\
\text { CDMX - Monterrey } \\
\text { CDMX - Tijuana }\end{array}$ & Perú & $\begin{array}{l}\text { Lima - Arequipa } \\
\text { Lima - Juliaca } \\
\text { Lima - Pucallpa } \\
\text { Lima - Iquitos } \\
\text { Lima - Cajamarca }\end{array}$ \\
\hline
\end{tabular}




\begin{tabular}{|c|c|c|c|}
\hline País & Rutas & País & Rutas \\
\hline EE.UU. & $\begin{array}{l}\text { Miami - Orlando } \\
\text { Miami - Atlanta } \\
\text { San Francisco - Los Ángeles } \\
\text { San Francisco - Las Vegas } \\
\text { Los Ángeles - Nueva York } \\
\text { Chicago - Nueva York }\end{array}$ & Brasil & $\begin{array}{l}\text { Rio de Janeiro - Brasilia } \\
\text { Rio de Janeiro - Sao Paulo } \\
\text { Brasilia - Sao Paulo } \\
\text { Brasilia - Belo Horizonte } \\
\text { Sao Paulo - Belo Horizonte }\end{array}$ \\
\hline Canadá & $\begin{array}{l}\text { Toronto - Quebec } \\
\text { Toronto - Halifax } \\
\text { Toronto - Winniepeg } \\
\text { Toronto - Vancouver } \\
\text { Toronto - Calgary } \\
\text { Toronto - Ottawa } \\
\text { Toronto - Montreal }\end{array}$ & España & $\begin{array}{l}\text { Madrid - Barcelona } \\
\text { Madrid - La Coruña } \\
\text { Barcelona - Palma de Mallorca } \\
\text { Barcelona - Málaga } \\
\text { Madrid - Ibiza }\end{array}$ \\
\hline Argentina & $\begin{array}{l}\text { Buenos Aires - Córdoba } \\
\text { Buenos Aires - Mendoza } \\
\text { Buenos Aires - Salta } \\
\text { Buenos Aires - Bariloche } \\
\text { Buenos Aires - Iguazú } \\
\text { Buenos Aires - Rosario }\end{array}$ & Francia & $\begin{array}{l}\text { París - Toulouse } \\
\text { París - Nantes } \\
\text { París - Ajaccio } \\
\text { París - Lyon } \\
\text { París - Niza }\end{array}$ \\
\hline
\end{tabular}

Fuente: el investigador

También se calculó la relación tasas e impuestos contra el precio del boleto, para observar el peso que tienen los tributos en el pago que deben hacer los pasajeros. Además de lo anterior, se aprovechó la data recabada para observar la relación entre las aerolíneas dominantes por cada ruta y el rango de sus precios.

\section{Resultados y discusión}

\subsection{Mercado Colombiano}

En el mercado colombiano se tomaron seis rutas troncales, principales o que de alguna manera revisten particular importancia para el transporte doméstico. Como se puede apreciar en la tabla 2, las aerolíneas que interactúan son Avianca, Latam y Viva Air; tres de las seis rutas son operadas por las tres aerolíneas y las otras tres rutas solo operan Avianca y Latam. En las seis rutas, la aerolínea dominante, es decir, la que acapara el mayor número de frecuencias, es Avianca con un 68,59\%, seguida por Latam con un 23,08\% y Viva Air con un $8,33 \%$. 
Tabla 2. Participación de las aerolíneas por cada ruta. Mercado Colombiano

\begin{tabular}{|c|c|c|c|}
\hline Ruta & Aerolíneas & Frecuencias & Porción de mercado \\
\hline \multirow{3}{*}{ Bogotá - Medellín } & Avianca & 30 & $60,00 \%$ \\
\cline { 2 - 4 } & Viva Air & 9 & $18,00 \%$ \\
\cline { 2 - 4 } & Latam & 11 & $22,00 \%$ \\
\hline \multirow{2}{*}{ Bogotá - Cali } & Avianca & 23 & $71,88 \%$ \\
\cline { 2 - 4 } & Latam & 9 & $28,13 \%$ \\
\hline \multirow{2}{*}{ Bogotá - Cúcuta } & Avianca & 6 & $85,71 \%$ \\
\cline { 2 - 4 } & Latam & 1 & $14,29 \%$ \\
\hline \multirow{3}{*}{ Bogotá - Barranquilla } & Latam & 4 & $19,05 \%$ \\
\cline { 2 - 4 } & Avianca & 17 & $80,95 \%$ \\
\hline \multirow{3}{*}{ Bogotá - Bucaramanga } & Latam & 8 & $26,67 \%$ \\
\cline { 2 - 4 } & Avianca & 19 & $63,33 \%$ \\
\cline { 2 - 4 } & Viva Air & 3 & $10,00 \%$ \\
\cline { 2 - 4 } & Viva Air & 1 & $6,25 \%$ \\
\cline { 2 - 4 } & Latam & 3 & $18,75 \%$ \\
\hline \multirow{3}{*}{ TOTAL } & Avianca & 12 & $75,00 \%$ \\
\cline { 2 - 4 } & Avianca & 107 & $68,59 \%$ \\
\cline { 2 - 4 } & Viva Air & 13 & $8,33 \%$ \\
\hline & Latam & 36 & $23,08 \%$ \\
\hline
\end{tabular}

Fuente: datos obtenidos de (Despegar Colombia S.A.S, 2018), cálculos del investigador

En este análisis de las rutas troncales se observa una mayor concentración que la indicada por Aerocivil (2018) para todo el mercado colombiano, en el que Avianca posee el 53,40\%, mientras para las rutas troncales su participación aumenta al $68,59 \%$. Esto puede obedecer a la mayor rentabilidad que ofrecen las rutas principales, donde se desplazan una cantidad superior de pasajeros, lo cual las hace más atractivas para las líneas aéreas, además que se adaptan mejor al tipo de aeronaves que poseen estas aerolíneas.

En este mismo orden de ideas, se calculó el índice Herfindahl-Hirschman (IHH) por cada ruta, en la tabla 3 se observa este índice y el precio promedio por kilómetro de cada ruta, así como el coeficiente de correlación entre estas dos variables.

Tabla 3. Correlación IHH - Precio promedio/Km

\begin{tabular}{|c|c|c|}
\hline Ruta & IHH & $\begin{array}{c}\text { Precio Promedio } \\
\text { (COP) } / \mathbf{K m}\end{array}$ \\
\hline Bogotá - Medellín & 4216 & 1035 \\
\hline Bogotá - Cali & 6517 & 763 \\
\hline Bogotá - Cúcuta & 7387 & 509 \\
\hline Bogotá - Barranquilla & 7402 & 216 \\
\hline Bogotá - Cartagena & 4555 & 244 \\
\hline Bogotá - Bucaramanga & 5977 & 689 \\
\hline Coeficiente de Correlación & \multicolumn{2}{|c|}{} \\
\hline
\end{tabular}

Fuente: El investigador

El índice de concentración va desde 4.216 en la ruta Bogotá - Medellín, hasta 7.402 en la ruta Bogotá Barranquilla, es decir, todas las rutas están por encima del valor de 1.800, lo cual indica que existe una alta concentración. Se observa una correlación negativa entre las dos variables, por lo que, a medida que aumenta la concentración en la ruta, el precio promedio por kilómetro disminuye, pero también hay que destacar que la correlación es mínima, ya que se ubica en un -0,39.

Contrariamente a lo anterior, cuando se correlaciona el IHH con el precio mínimo por kilómetro, lo cual se presenta en la tabla 4, la correspondencia es positiva, con un valor de 0,32. A medida que aumenta la 
concentración, el precio mínimo también aumenta. En este caso también hay que observar que la correlación es mínima.

Tabla 4. Correlación IHH - Precio mínimo/Km

\begin{tabular}{|c|c|c|}
\hline Ruta & IHH & $\begin{array}{c}\text { Precio Mínimo } \\
(\mathbf{C O P}) / \mathbf{K m}\end{array}$ \\
\hline Bogotá - Medellín & 4216 & 299 \\
\hline Bogotá - Cali & 6517 & 554 \\
\hline Bogotá - Cúcuta & 7387 & 463 \\
\hline Bogotá - Barranquilla & 7402 & 140 \\
\hline Bogotá - Cartagena & 4555 & 119 \\
\hline Bogotá - Bucaramanga & 5977 & 170 \\
\hline Coeficiente de Correlación & \multicolumn{2}{|c|}{$\mathbf{0 , 3 2}$} \\
\hline
\end{tabular}

Fuente: El investigador

Con la alta concentración en el mercado colombiano que posee la aerolínea Avianca, abría de esperarse que hiciera mayor uso de su poder de mercado y la correlación entre IHH y precio de los boletos aéreos fuera mayor, pero por el contrario esa correlación es baja para el caso de los precios mínimos o inversa para el precio promedio, lo cual hace entrever un manejo prudente de la aerolínea, pensando en el cuido de su cuota de mercado en el largo plazo.

\subsection{Comparación con otros mercados domésticos}

Al tomar una muestra de diez países y comparar los precios de los boletos/Km se observan los resultados en la tabla 5 y la gráfica 1 :

Tabla 5. Índice Herfindahl-Hirschman por país

\begin{tabular}{|c|c|c|c|}
\hline País & IHH & País & IHH \\
\hline EEUU & 3283 & Colombia & 5637 \\
\hline México & 4057 & Chile & 5641 \\
\hline Brasil & 4783 & Canadá & 5706 \\
\hline Perú & 4991 & Argentina & 6372 \\
\hline España & 4992 & Francia & 9559 \\
\hline \multicolumn{4}{|c|}{ Fuente: El investigador }
\end{tabular}

Se observa que Colombia se encuentra en un nivel intermedio entre la muestra con un valor de 5.637; así mismo se visualiza que todos los países, incluyendo EEUU que posee el menor IHH con un valor de 3.282, están por encima de 1.800, lo cual establece una alta concentración en todos los mercados domésticos.

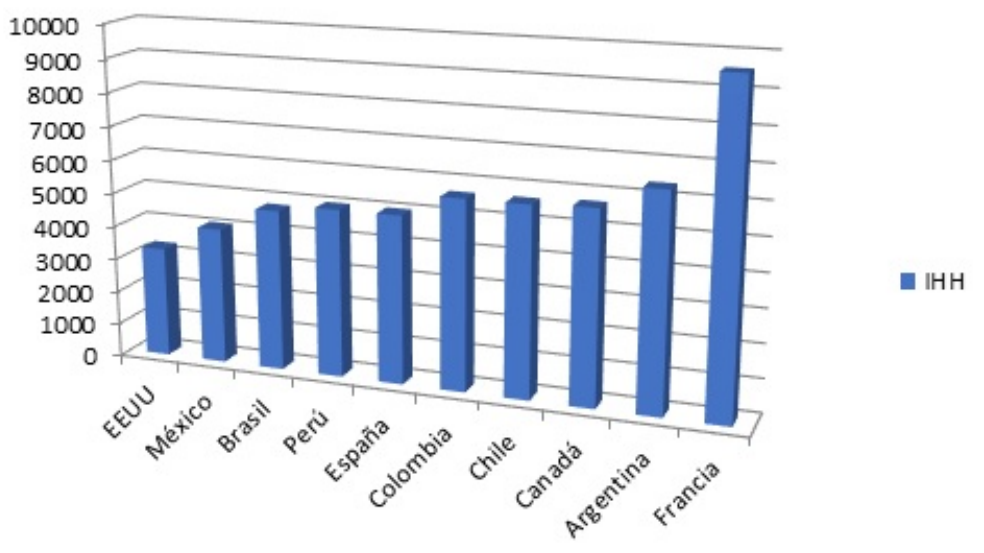

Gráfico 1. Índice Herfindahl-Hirschman por cada país

Fuente: El Investigador 
Destaca el IHH de Francia, el cual está en un valor de 9.559 puntos, cercano al máximo de 10.000, lo cual indicaría una concentración total del mercado doméstico. Pero estos datos es necesario analizarlos en conjunto con los precios/Km de cada mercado, los cuales se pueden visualizar en la tabla 6 y gráfico 2 .

Tabla 6. Indicador Precio Boleto (COP)/Km por cada país

\begin{tabular}{|c|c|c|c|}
\hline País & $\begin{array}{c}\text { Precio Boleto } \\
(\mathbf{C O P}) / \mathbf{K m}\end{array}$ & País & $\begin{array}{c}\text { Precio Boleto } \\
(\mathbf{C O P}) / \mathbf{K m}\end{array}$ \\
\hline Argentina & 378 & Colombia & 625 \\
\hline México & 395 & Canadá & 709 \\
\hline Perú & 406 & España & 911 \\
\hline Chile & 533 & Brasil & 1118 \\
\hline EEUU & 567 & Francia & 1476 \\
\hline
\end{tabular}

Fuente: El Investigador

Al comparar el índice de concentración con los precios de boletos por kilómetro recorrido, se puede observar que EEUU posee la menor concentración de todos los países estudiados, pero en el precio/Km se ubica en la parte intermedia de la tabla; Colombia está de quinto lugar entre los países con mayor concentración y permanece en el quinto lugar en el indicador de precio/Km. Brasil, de ser el tercer país con menor concentración, pasa a ser el segundo país con el precio/Km más alto. Francia es el país con la mayor concentración y recurrentemente también es el de mayor precio/Km. Argentina es el país con segunda mayor concentración, pero es el que reporta menor precio/Km.

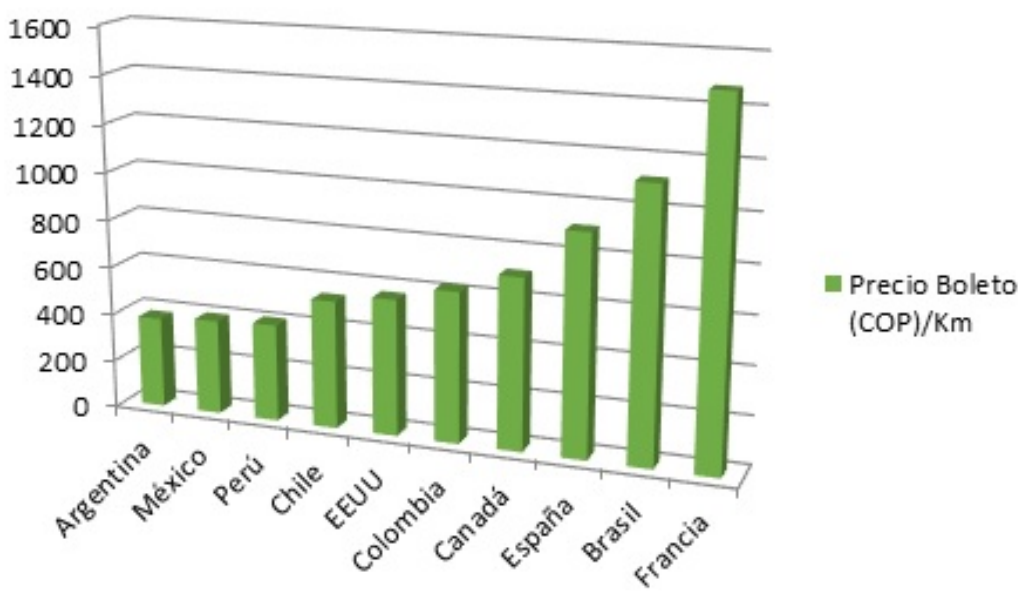

Gráfica 2. Relación precio del boleto/Km

Fuente: El Investigador

Para complementar los datos anteriores, se realizó el análisis de correlación entre las variables IHH y Precio boleto / Km, para ello se presenta la información de la tabla 7. El índice de correlación entre las variables IHH y Precio boleto / Km se encuentra en un valor de 0,63; este dato indica que existe una correlación regular entre estas variables, lo cual es bastante significativo, a medida que la concentración en los mercados domésticos aumenta, también lo hacen los precios de los boletos. 
Tabla 7. Correlación IHH - Precio boleto (COP) / Km

\begin{tabular}{|c|c|c|}
\hline País & IHH & Precio Boleto $(\mathbf{C O P}) / \mathbf{K m}$ \\
\hline EEUU & 3283 & 567 \\
\hline México & 4057 & 395 \\
\hline Brasil & 4783 & 1118 \\
\hline Perú & 4991 & 592 \\
\hline España & 4992 & 911 \\
\hline Colombia & 5637 & 625 \\
\hline Chile & 5641 & 598 \\
\hline Canadá & 5706 & 709 \\
\hline Argentina & 6372 & 378 \\
\hline Francia & 9559 & 1476 \\
\hline Coeficiente de correlación & \multicolumn{2}{|c|}{} \\
\hline
\end{tabular}

Estos resultados demuestran que efectivamente existe una influencia de las empresas que tienen acaparado mayor cantidad de mercado; mientras el índice HH es más alto, significa que existen aerolíneas que poseen mayor cantidad de mercado, ejercen ese poder y con base a ello fijan las tarifas al alza.

Los elementos de mayor dispersión, como es el caso de Argentina, responden a otras realidades o circunstancias puntuales, ya que la aerolínea dominante en ese país es Aerolíneas Argentina, la cual pertenece al estado argentino, y es posible que su principal objetivo no sea aprovecharse de su dominio de mercado y maximizar sus ganancias financieras, sino sus prioridades pueden ser más de orden estratégico y social para el estado. En este sentido, Eleisegui (2018) afirma que Aerolíneas Argentinas comenzó en agosto de 2018 distintas campañas promocionales, las cuales incluyen descuentos hasta de $50 \%$ del valor del boleto, esto para competir con las aerolíneas de bajo costo y tomando en consideración la baja del poder adquisitivo de la población argentina.

Por otro lado, existe otro factor bastante importante para los usuarios de las aerolíneas, que incide de manera considerable en el precio total que cualquier pasajero debe pagar en un vuelo doméstico; este factor corresponde a los impuestos y tasas. Para destacar el peso de este factor se presenta la relación impuestos y tasas como porcentaje del precio total del boleto aéreo normalizado por la cantidad de kilómetros de las rutas aéreas. En la tabla 8 y la gráfica 4 se refleja esta relación, en la cual Colombia sigue estando entre los valores intermedios, cercano más a los valores más altos.

Tabla 8. Relación impuestos y tasas sobre el precio del boleto por kilómetro

\begin{tabular}{|c|c|c|c|}
\hline País & Relación imp./Boleto & País & Relación imp./Boleto \\
\hline Brasil & $3,93 \%$ & España & $16,59 \%$ \\
\hline Chile & $6,55 \%$ & Colombia & $20,59 \%$ \\
\hline EEUU & $14,39 \%$ & Canadá & $22,44 \%$ \\
\hline Argentina & $15,16 \%$ & Perú & $24,66 \%$ \\
\hline Francia & $15,47 \%$ & México & $31,88 \%$ \\
\hline
\end{tabular}

Este indicador posee para cada uno de los países estudiados valores bastante dispersos, que van desde 3,93\% en Brasil, hasta 31,88 \% en México. Estos dos países son de los que poseen las rutas más largas, varias por encima de los $1.000 \mathrm{Kms}$, lo cual hace ver que el número de kilómetros por ruta no es determinante en el indicador presentado. Brasil y Chile destacan por la relación más baja; Colombia tiene un 20,59\%, entendiéndose que las tasas impositivas para el sector aéreo son bastante altas en el país. A medida que el porcentaje de impuestos y tasas aumenta, por supuesto que aumenta el precio total que paga cada pasajero 
y por lo tanto este servicio puede hacerse prohibitivo para algunos usuarios, esto independientemente de que esos recursos se utilicen para mantener una infraestructura aeroportuaria en condiciones óptimas.

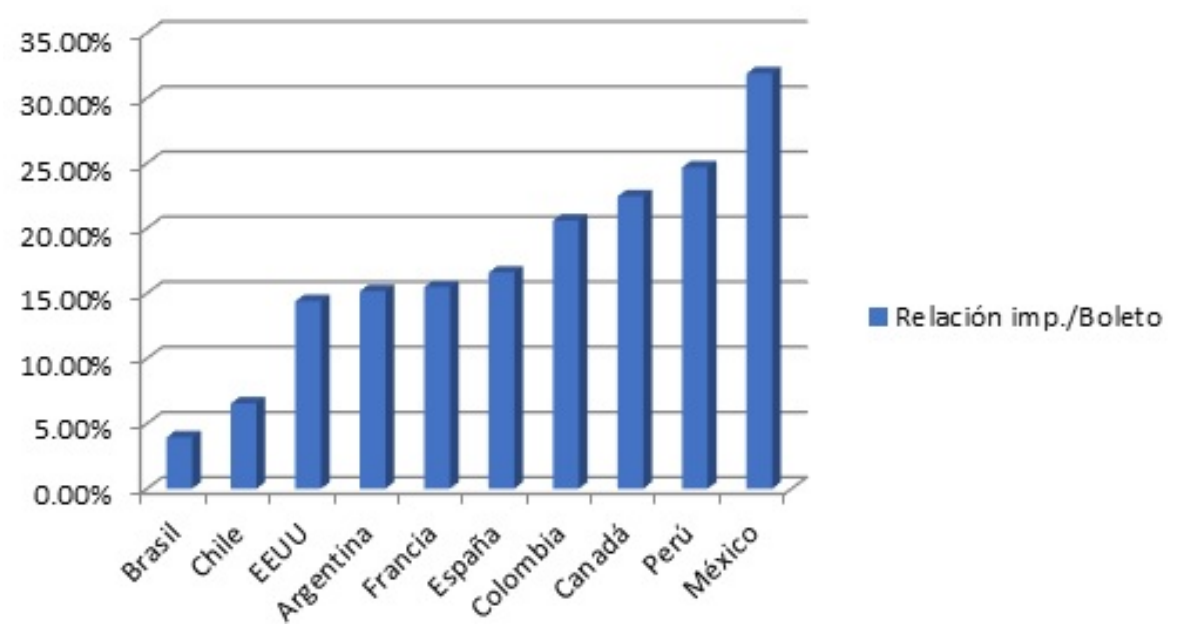

Gráfico 3. Relación impuestos y tasas por el valor total del boleto/Km Fuente: El Investigador

Como lo refieren Cristóbal, Panchana, Tomalá y Asencio (2017), si bien los impuestos son necesarios para los gastos del estado, entre ellos se encuentran la infraestructura aeroportuaria y la seguridad de la misma, una alta tasa de estos impuestos puede aumentar significativamente el desembolso que debe hacer el consumidor por los productos y servicios a los cuales se aplica, haciendo menos competitivo el mercado nacional frente a otros, esto tomando en consideración los bienes transables o que tengan productos substitutos; el transporte aéreo posee estas condiciones siempre y cuando el viajero tenga la libertad de elegir su destino dependiendo de lo económico que resulte el mismo, esto aplica para viajeros turistas, mas no para viajeros de negocios.

\section{Conclusiones}

El mercado doméstico del transporte aéreo en Colombia está abarcado, en sus rutas troncales, por tres aerolíneas, de las cuales Avianca ha sido la de mayor trayectoria y en la actualidad es la que acapara el mayor número de frecuencias con un 68,59\% del total. El índice HH, utilizado como medida de concentración, evidencia que el mercado colombiano sobrepasa el umbral de los 1.800 puntos, por encima del cual se considera una concentración alta.

$\mathrm{Al}$ relacionar el índice $\mathrm{HH}$ de cada ruta estudiada con el precio/Km se observa una correlación mínima, con lo cual no se puede concluir de manera absoluta en este aspecto. El índice HH para Colombia arrojó un valor de 5.549, que al compararlo con el índice HH de los demás mercados en estudio, se establece una posición intermedia; así mismo cuando se observa el precio del boleto por kilómetro de ruta, Colombia ocupa una posición media dentro del grupo de países.

El transporte aéreo ha sido un mercado históricamente inestable, muchas aerolíneas, en cualquier parte del mundo, han entrado en procesos de quiebra luego de algunos períodos de bonanza; variables como el crecimiento económico o el precio del petróleo, entre otras, afectan enormemente el desempeño financiero de las aerolíneas. Esto ha estado previsto desde los inicios de la aviación, por ello en los tratados primigenios como el de Varsovia en 1.929 o el Convenio de Chicago en 1.947 se ha tratado de establecer un equilibrio en los mercados aéreos, que la misma oferta y demanda no ha podido conseguir, esto debido a distintos factores como las barreras de entrada al negocio y el tamaño reducido de los mismos mercados. Por esto, si bien es cierto que la totalidad de los mercados estudiados rebasan por mucho el umbral de los 1.800 puntos del 
índice HH para determinar la concentración de un sector, también es cierto que en Colombia se observa un equilibrio logrado con base a vigilancia de las autoridades competentes y pareciera también, cierto accionar lógico y visualización del largo plazo de parte de las aerolíneas.

La competencia en cualquier mercado es importante desde el punto de vista social debido a que con ella se pueden alcanzar los mejores precios para los usuarios y desde el punto de vista económico las empresas involucradas se esmeran por alcanzar eficiencias cada vez mayores. Pero al no poderse lograr los niveles de competencia ideales, lo cual debería redundar en variables fundamentales como el precio, entonces se debe tratar de lograr por otras vías. En el mercado colombiano, estos procesos vendrían dándose desde 2012, momento en que se les otorga a las aerolíneas la libertad para fijar sus tarifas, pero siempre bajo la vigilancia de la Aeronáutica Civil, autoridad encargada del sector aéreo en el país. A esto se le debe agregar un proceder prudente y estratégico en las empresas de transporte aéreo, que han sabido equilibrar estas condiciones con su pensamiento de largo plazo, entendiendo que lo sano para el negocio es el equilibrio.

Las iniciativas legislativas que buscan regular el mercado del transporte aéreo en Colombia deben estudiarse en profundidad y analizar las afectaciones que pudieran tener; estas regulaciones de tarifas podrían influir en la iniciativa empresarial y el grado de inversión de las aerolíneas para captar más pasajeros y en el largo plazo podría incidir negativamente en los niveles de calidad de servicio en el mercado.

Por último, la correspondencia de los impuestos y tasas respecto al valor total del boleto refleja para Colombia una relación más cercana a los mayores valores encontrados. Ciertamente es un dato relativo y será aprobado por los usuarios y justificados estos desembolsos en la medida en que los servicios aeroportuarios reflejen los niveles de seguridad, confort y adaptación a las nuevas tendencias internacionales. Pero en definitiva, estos impuestos y tasas incrementan considerablemente el costo final del pasaje aéreo, lo que puede influir negativamente en sectores económicos tan importantes en Colombia como el turismo.

Ha sido importante la utilización del Índice Herfindahl-Hirschman ya que no hay evidencia de que el mismo haya sido aplicado al mercado de transporte aéreo, lo cual constituye una novedad de esta investigación. En una segunda etapa de la investigación se ampliará la muestra de los países en estudio, así mismo se utilizará otro indicador de concentración o desequilibrio en los mercados además del IHH.

\section{Referencias}

[1] Aerocivil. (2012). Resolución 904. Bogotá, Colombia: Imprenta Nacional.

[2] Aerocivil. (2018). Estadísticas de las actividades aeronáuticas. Recuperado el 09 de 10 de 2018, de Aerocivil: http://www.aerocivil.gov.co/atencion/estadisticas-de-las-actividades-aeronauticas/boletines-operacionales

[3] Betancor, O., Gallego, A., \& González, M. J. (2013). Observatorio de Transporte Aéreo, Las tarifas en el mercado español de transporte aéreo. Fundación de Estudios de Economía Aplicada.

[4] Cardoso, C. (2016). Competencia económica en el sector de transporte aéreo de pasajeros en México. Economía Informa (397), 44. https://doi.org/10.1016/j.ecin.2016.03.003

[5] Castañeda, C. (2007). Indicadores de Concentración: Una revisión del marco conceptual y la experiencia internacional. Osiptel, 5.

[6] Cristóbal, N.; Panchana, M; Tomalá, M. y Asencio, L. (2017). Las reformas al impuesto al valor agregado y su efecto en la economía del consumidor en la provincia de Santa Elena. Revista Ciencia y Tecnología OPSE, 4(1). DOI: https://doi.org/10.26423/rctu.v4i1.255

[7] Cuevas, M. (2009). Las condiciones de competencia en las principales rutas de aerolíneas nacionales e internacionales, y los mercados domésticos en cada país del Istmo Centroamericano. México, D.F.: Naciones Unidas.

[8] Despegar Colombia S.A.S. (2018). Despegar Colombia. Recuperado el 2018, de https://www.despegar.com.co/ 
[9] DICES (2018). Distancias entre aeropuertos. Recuperado de: https://www.dices.net/aeropuertos/ distancias.html

[10] Galbiati, J. (2016). Conceptos básicos de estadísticas. Buenos Aires: Brown.

[11] Gutierrez, J., Zamudio, N. (2008). Medidas de Concentracion y Competencia. Reporte de estabilidad financiera, 4. https://doi.org/10.32468/tef.29

[12] Hernández, R., Collado, C., Baptista, P. (2014). Metodología de la investigación. México: Mc-Graw Hill.

[13] Hernández, R. y Mendoza, C. (2018). Metodología de la investigación: las rutas cuantitativa, cualitativa y mixta. México: Mc-Graw Hill.

[14] Lind, D. (2015). Estadística aplicada a los negocios y a la economía. (16a. ed.). McGraw-Hill Interamericana.

[15] Martínez, C. (2012). Estadística básica aplicada (4a. ed.). Bogotá: Ecoe Ediciones.

[16] Méndez, J. (2014). Fundamentos de economía. Página 291. (6ta ed.). México: McGraw-Hill Interamericana.

[17] Nauffal, S. (2007). Organización industrial y competencia estratégica de las aerolíneas en Colombia (Tesis de pregrado). Bogotá: Pontificia Universidad Javeriana.

[18] Novelo, F. (2015). Análisis crítico del libro Mas allá de la mano invisible. Fundamentos para una nueva economía política. Argumentos (México, D.F.), 28(77), 245-251. Recuperado de: http://www.scielo.org.mx/scielo.php? script=sci_arttext\&pid=S0187-57952015000100012

[19] Oficina de Regulación Económica. (2009). Revisión y análisis de la estructura de costos de transporte aéreo. Bogotá.

[20] Ortega, A. (2011). Economía colombiana (4a. ed.). Bogotá: Ecoe Ediciones.

[21] Pabón, A., Henao, Y., Rodriguez, J., Campo, J., Herrera, J. (2015). Una visión general del transporte aéreo en Colombia. N 11. Bogotá: Superintendencia de Industria y Comercio.

[22] Pinzón, J. (2011). La Concentración y el Precio de los Servicios Financieros en Colombia (Trabajo de Investigación). Obtenido de Universidad Nacional de Colombia: http://bdigital.unal.edu.co/5140/1/La_ ConcentraciC3B3n_y_el_precio_de_los_servicios_financieros_en_Colombia_1989-2008.pdf

[23] Rodríguez, E. (2012). Toma de decisiones: la economía conductual. Repositorio intitucional Universidad de Oviedo. Recuperado de: http://hdl.handle.net/10651/13074

[24] Semana. (2018). Pasajeros podrán reclamar hasta 60 por ciento de los tiquetes por retrasos en vuelos. Entrevista al congresista Favio Arroyave. Recuperado de: https://www.semana.com/nacion/articulo/ pasajeros-podran-reclamar-hasta-60-de-los-tiquetes-

[25] Veiga, J., De la Fuente, E., Zimmermann, M. (2008). Modelos de estudios en investigación aplicada: conceptos y criterios para el diseño. Medicina y Seguridad del Trabajo. https://doi.org/10.4321/ s0465-546x2008000100011 